Original Research

\title{
Relativistic Wigner Function for Quantum Walks
}

Fabrice Debbasch $^{*}$

Sorbonne Universit'e, Observatoire de Paris, Universit'e PSL, CNRS, LERMA, F-75005, Paris, France;

E-Mail: fabrice.debbasch@gmail.com

* Correspondence: Fabrice Debbasch; E-Mail: fabrice.debbasch@gmail.com

Academic Editors: Hossein Hosseinkhani and Sotirios Baskoutas

Special Issue: Quantum Confinement Effects in Nano Material

Recent Progress in Materials

2020, volume 2 , issue 1

doi:10.21926/rpm.2001002
Received: October 15, 2019

Accepted: December 08, 2019

Published: January 06, 2020

\begin{abstract}
A relativistic Wigner function for free Discrete Time Quantum Walks (DTQWs) on the square $2 D$ space-time lattice is defined. The transport equation obeyed by the relativistic Wigner function is derived in terms of discrete derivatives and degenerates in the continuum limit into the transport equation obeyed by the Wigner function of 2D Dirac fermions. The first corrections to the continuous equation induced by the discreteness of the lattice are also determined and the similarities with non-quantum relativistic transport in phase space are discussed further.
\end{abstract}

\section{Keywords}

Discrete time quantum walks; relativistic phase-space; relativistic transport equation

\section{Introduction}

Discrete Time Quantum Walks (DTQWs) are unitary quantum automata that can be viewed as the formal generalizations of classical random walks. Following the seminal work of Feynman [1],

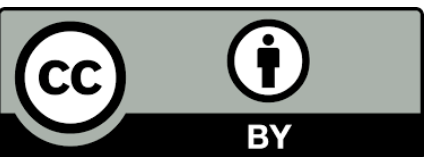

(C) 2020 by the author. This is an open access article distributed under the conditions of the Creative Commons by Attribution License, which permits unrestricted use, distribution, and reproduction in any medium or format, provided the original work is correctly cited. 
GrÖssing and Zeilinger [2] and Aharonov [3], they were considered in a systematic way by Meyer [4]. DTQWs have been realized experimentally with a wide range of physical objects and setups [511] which include quantum dots [12] and silicon nano-photonic chips [13]. DTQWs are studied in several diverse of contexts, ranging from quantum optics [14] to quantum algorithmics [15, 16], condensed matter physics [17-21], including transport in graphene [22-24], hydrodynamics [25] and biophysics [26, 27].

It is well known that several DTQWs can be viewed as discrete versions of the continuous Dirac and Weyl fermion dynamics [28-35]. These DTQWs have a continuum limit which coincides with the Dirac equation and they even display exact discrete gauge invariance properties [36-40].

However, the phase-space behavior of these DTQWs still remains unknown, which could be investigated well with the help of Wigner functions. Due to several fundamental and practical reasons, building Wigner functions for DTQWs is indeed an interesting problem. Quite generally speaking, there are essentially three possible logically complete formulations of quantum mechanics. Formulation 1 is the canonical quantization. It starts by considering position and momentum as operators which do not commute. The commutation relation is then realized by choosing position and momentum to be operators acting on time-dependent wave-functions defined over physical space and the time-evolution on the wave-function is fixed by the Hamiltonian. Formulation 2 is based on Feynman's path integral, which attributes to each possible path between an initial state and a final state with a certain probability amplitude defined, at least formally, in terms of the classical i.e. non-quantum action of the system. Formulation 3 is based on the Wigner function [41], which is a quantum generalization of classical phase-space densities and whose time-evolution is fixed by its Moyal bracket $[41,42]$ with the Hamiltonian.

DTQWs are historically connected to Formulation 2 because they originate in Feynman's attempts to find a suitable discretization to compute path-integrals for fermions. DTQWs also connect with Formulation 1, because they can be viewed as wave-functions defined over discrete space-time and evolving unitarily under the action of their so-called pseudo-Hamiltonian. However, the connection with Formulation 3 has not been established. Indeed, even if Wigner functions have already been considered for Dirac quantum walks [43-46], the proposed definitions are not manifestly covariant and do not coincide in the continuum limit, with the standard, manifestly covariant Wigner functions for Dirac fermions [47-49]. Nor does their evolution equation coincides, in the continuum limit, with the usual phase-space transport equation for Dirac fermions.

Moreover, defining Wigner functions for DTQWs is not a purely academic topic. For example, interesting results on trapped non-relativistic fermions obtained recently in terms of the nonrelativistic Wigner function [50] suggest that a relativistic Wigner function is the proper tool to study confinement of Dirac fermions and Dirac quantum walks.

More generally, the Wigner function of a quantum system can be obtained by Fourier transformation of the space-time representation of the density operator of this system with respect to one of its variables. Wigner functions can thus be used to study how density operators evolve in time and, in particular, to follow their time-evolutions in the presence of noise. Working with Wigner functions is actually often easier than working with density operators as using phasespace is rather intuitive. For example, Wigner functions are usually the best way to write down and study the Master equations describing decoherence, if the classical limit of the transport equation coincides with standard Kolmogorov equations describing phase-space transport in stochastic non-quantum systems. Owing to the relevance of both DTQWs and decoherence to 
quantum information, constructing Wigner functions for DTQWs and determining their evolution equations are thus important issues in the field.

The aim of this article is to define a relativistic Wigner function for free DTQWs in flat 2D spacetime. We first extend continuous distribution theory to analyze a discrete lattice. We then define a discrete relativistic Wigner function for DTQWs and derive the corresponding discrete relativistic transport equation, which we express in terms of discrete analogs to usual continuous derivatives. In the continuum limit, the discrete Wigner function tends towards the Wigner function of Dirac particles and the transport equation tends toward the equation obeyed by the Wigner function of Dirac fermions in continuous space-time. We also suggest the first correction to the transport equation induced by the discreteness of the lattice on which the DTQWs propagate and finally discuss the results in the last section.

\section{A Simple Dirac QW}

We work with two-component wave-functions $\Psi$ defined in $2 D$ discrete space-time where

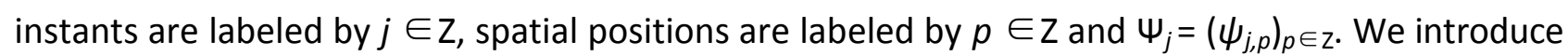
a basis $\left(b_{A}\right)=\left(b_{L}, b_{R}\right)$ in Hilbertspace space and the components $\Psi^{A}=\left(\Psi^{L}, \Psi^{R}\right)$ of the arbitrary wave-function $\Psi$ in this basis. The Hilbert product is defined by $\left\langle\psi, \varphi>=\sum_{A, j, p}\left(\psi^{A}\right)_{j, p}^{*}\left(\varphi^{A}\right)_{j, p}\right.$, which makes the basis $\left(b_{A}\right)$ orthonormal. Consider now the quantum walk $\Psi_{j+1}=U_{j} T \Psi_{j}$ where $T$ is the spatial-translation operator defined by $\left(T \Psi_{j}\right)_{j, p}=\left(\Psi_{j, p+1}^{L}, \Psi_{j, p-1}^{R}\right)^{T}$ and $U_{j}$ is an $S U(2)$ operator defined by $\left(U_{j} \Psi_{j}\right)_{j, p}=U(\vartheta) \Psi_{j, p}(1)$ where $U(\vartheta)=\left(\begin{array}{cc}\cos \theta & -i \sin \theta \\ -i \sin \theta & \cos \theta\end{array}\right), \quad$ (2) with constant $\vartheta$. It was shown earlier [38] that this quantum walk, in the continuum limit defined by $t_{j}=j \epsilon, x_{p}=p \epsilon$, $\vartheta=\epsilon m$, tends to the Dirac equation for a 2D spinor of mass $m$ in the flat Minkovski space-time with coordinates $(t, x)$ as $\epsilon$ tends to zero.

\section{Basic Tools}

\subsection{Discrete Derivatives}

Let us now rewrite the above equations with the help of discrete covariant derivative. We define:

$$
\begin{gathered}
\left(D_{j} f\right)_{j, p}=\frac{1}{2}\left(f_{j+1, p}-f_{j-1, p}\right) \\
\left(D_{j j} f\right)_{j, p}=\frac{1}{2}\left(f_{j+1, p}+f_{j-1, p}-2 f_{j, p}\right) \\
\left(D_{p} f\right)_{j, p}=\frac{1}{2}\left(f_{j, p+1}-f_{j, p-1}\right) \\
\left(D_{p p} f\right)_{j, p}=\frac{1}{2}\left(f_{j, p+1}+f_{j, p-1}-2 f_{j, p}\right)(3), \text { where } f \text { is an }
\end{gathered}
$$

arbitrary $j$ - and $p$-dependent quantity. These are discrete versions of the usual partial derivatives.

Inverting the above equations delivers:

$$
\begin{gathered}
f_{j+1, p}=f_{j, p}+\left(D_{j} f\right)_{j, p}+\left(D_{j j} f\right)_{j, p} \\
f_{j-1, p}=f_{j, p}-\left(D_{j} f\right)_{j, p}+\left(D_{j j} f\right)_{j, p} \\
f_{j, p+1}=f_{j, p}+\left(D_{p} f\right)_{j, p}+\left(D_{p p} f\right)_{j, p} \\
f_{j, p-1}=f_{j, p}-\left(D_{p} f\right)_{j, p}+\left(D_{p p} f\right)_{j, p}(4) .
\end{gathered}
$$


Let us now rewrite the equation of motion of the walk, $\Psi_{j+1}=U_{j} T \Psi_{j}$, in terms of the above discrete derivatives. One starts by subtracting $\Psi_{j}$ from both sides of the equation, getting $\Psi_{j+1}-\Psi_{j}$ $=\left(U_{j} T-1\right) \Psi_{j}$, (5) which can be rewritten as $\left(D_{j} \Psi\right)_{j, p}+\left(D_{j j} \Psi\right)_{j, p}=\left(\left(U_{j} T-1\right) \Psi_{j}\right)_{j, p}(6)$, or, equivalently, $\left(D_{j} \Psi\right)_{j, p}=\left(\left(U_{j} T-1\right) \Psi_{j}\right)_{j, p}-\left(D_{j j} \Psi\right)_{j, p .}(7)$.

Let us now express $T \Psi_{j}$ in terms of the discrete derivatives. Since $\left(T \Psi_{j}\right)_{j, p}^{L}=\left(\Psi_{j}\right)_{j, p+1}^{L}$, one can write $\left(T \Psi_{j}\right)_{j, p}^{L}=\left(\Psi_{j}\right)^{L}+\left(D_{p} \Psi_{j}^{L}\right)_{j, p}+\left(D_{p p} \Psi_{j}^{L}\right)_{j, p}$ (8). Similarly, $\left(T \Psi_{j}\right)_{j, p}^{R}=\left(\Psi_{j}\right)^{R}-\left(D_{p} \Psi_{j}^{R}\right)_{j, p}$ $+\left(D_{p p} \Psi_{j}^{R}\right)_{j, p}$ (9). It follows that $\left(\left(U_{j} T-1\right) \Psi_{j}\right)_{j, p}=\left(\left(U_{j}-1\right) \Psi_{j}\right)_{j, p}+\left(U_{j} \sigma_{3} D_{p} \Psi_{j}\right)_{j, p}+\left(U_{j} D_{p p} \Psi_{j}\right)_{j, p}$ (10), where $\sigma_{3}$ is the operator represented by the third Pauli matrix in the basis $\left(b_{A}\right)$ i.e. $\sigma_{3}$ is represented by the matrix diag $(1,-1)$ in the basis $\left(b_{A}\right)$. The equation of motion of the $\mathrm{QW}$ can thus be rewritten as:

$$
\left(D_{J} \Psi^{A}\right)_{j, p}=\left(U \sigma_{3}\right)_{B}^{A}\left(D_{p} \Psi^{B}\right)_{j, p}+(\mathrm{U}-1) \frac{A}{B} \Psi_{j, p}^{B}-\delta_{B}^{A}\left(D_{j j} \Psi^{B}\right)_{j, p}+U_{B}^{A}\left(D_{P P} \Psi^{B}\right)_{j, p} \text { (11). Where }
$$
(1) $\frac{A}{B}=\delta_{B}^{A}=1$ if $\mathrm{A}=\mathrm{B}$ and 0 otherwise. In the continuum limit, the left-hand side and the first term on the right-hand side deliver the differential terms in the Dirac equation, the second contribution to the right-hand side delivers the mass term while the other two terms vanish as they are of a higher order.

Let us finally mention the following identities, which will be used in the next sections:

$D_{j}(f g)=\left(D_{j} f\right) g+f\left(D_{j} g\right)+\left(D_{j} f\right)\left(D_{j j} g\right)+\left(D_{j j} f\right)\left(D_{j} g\right)(12), D_{p}(f g)=\left(D_{p} f\right) g+f\left(D_{p} g\right)+\left(D_{p} f\right)\left(D_{p p} g\right)+$ $\left(D_{p p} f\right)\left(D_{p} g\right)(13)$. Note that the terms which disappear in the continuum limit are of order of 3 , and not 2 in $\epsilon$.

\subsection{Discrete Distributions}

We are interested in defining a relativistic Wigner function for DTQWs. This will involve Fourier analysis of the density operator of the walk with respect to one of its time- and one of its spacevariables. The space-time grid is infinite in both time and space and there is no reason to suppose that the density operator of the walk vanishes when either of these variables, and especially the time variable, tends to be infinite. It follows that the standard discrete Fourier transform, which operates on a finite number of points, cannot a priori be used for the Fourier analysis of the density operator. A similar problem arises in continuous analysis, when one wants to perform the Fourier analysis of functions of a real variable which does not decrease sufficiently at infinity. In the continuous analysis, the standard remedy is to work with distributions. Therefore extend to the discrete setting to the usual continuous theory of distributions. In a case where the density operator does has compact support, the theory will give compatible with the usual harmonic analysis defined by discrete Fourier transforms. For the sake of simplicity, all ideas are now presented for functions and distributions of one single discrete variable $n \in Z$, which plays the role of $j$ or $p$. The extension of these two discrete variables used in the following sections is straightforward.

We introduce as test functions the space of all functions $h$ of $n$ which admit a discrete Fourier transform $\hat{h}$ defined by $\hat{h}(k)=\sum_{n} \exp (i k n) h_{n}$. (14). All these functions have compact support. The conjugate momentum $k$ takes value in the first Brillouin zone of the lattice i.e. $(-\pi, \pi)$. The inverse Fourier transform is thus defined by:

$$
h_{n}=\frac{1}{2 \pi} \int_{-\pi}^{\pi} \exp (-i k n) \hat{h}(k) d k(15) .
$$


Any function $f$ defined on $Z$ can now be considered a distribution acting on this space of test functions and we define $f(h)=\langle f, h\rangle_{n}=\sum_{n} f_{n} h_{n}$ (16). We now define the discrete Fourier transform $\hat{f}$ of the distribution $f$ by its action on functions on the variable $k$. Let $H$ be such a function. We define $\hat{f}(H)$ by

$$
\hat{f}(H)=\sum_{n} \frac{1}{2 \pi} \int_{-\pi}^{\pi} d k \exp (i k n) f_{n} H(k)
$$

$=<f, \hat{H}\rangle_{\mathrm{n}} \quad$ (17). Introducing the natural product in Fourier-space $\left\langle u, v>_{k}=\frac{1}{2 \pi} \int_{-\pi}^{\pi} d k u(k) v(k)(18)\right.$, the definition of $\hat{f}$ can be rewritten as $\left\langle\hat{f}, H>_{k}=<\right.$ $f, \hat{H}>_{n}$.

Consider the Fourier transform of the derivative $F=D_{n} f$.

$$
\begin{aligned}
<\hat{F}, H>_{k} & =<F, \hat{\mathrm{H}}>_{n} \\
& =\sum_{n} \frac{1}{2 \pi} \int_{-\pi}^{\pi} d k \exp (i k n) F_{n} H(k) \\
& =\sum_{n} \frac{1}{2 \pi} \int_{-\pi}^{\pi} d k \exp (i k n) \frac{1}{2}\left(f_{n+1}-f_{n-1}\right) H(k) \\
& =\sum_{n} \frac{1}{2 \pi} \int_{-\pi}^{\pi} d k \exp (i k n) \frac{1}{2}(\exp (-i k)-\exp (i k)) f_{n} H(k) \\
& =\sum_{n} \frac{1}{2 \pi} \int_{-\pi}^{\pi} d k \exp (i k n) f_{n}(-i \sin k) H(k) \\
& =<G, H>_{k} \quad(19), \text { where } G(k)=-i(\sin k) \hat{f}(k) . \text { Thus, } \hat{F}(k)=-i(\sin k) \hat{f}(k)(20) . \text { In the }
\end{aligned}
$$
continuum limit, the continuous position $N$ and wave-vector $K$ are related to $n$ and $k$ by $N=\epsilon n$ and $K=k / \epsilon$, so $(20)$ becomes $\epsilon \widehat{f}^{\prime}(K)=-i \sin (\epsilon K) \hat{f}(K)(21)$, where $f^{\prime}=d f / d N$. At orders 0 and $1,(21)$ reads simply $\widehat{f}^{\prime}(K)=-i K \hat{f}(K)(22)$, but one finds, for example at second order in $\epsilon$ :

$$
\widehat{f}^{\prime}(K)=-i K\left(1-\frac{\epsilon^{2} K^{2}}{3}\right) \hat{f}(K)(23) \text {. }
$$

\section{Discrete Wigner Function}

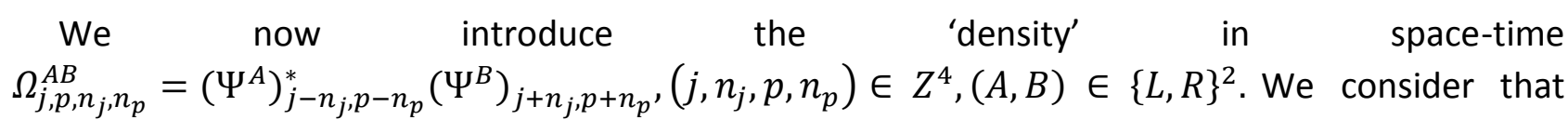
this object is, at fixed $(j, p)$, a discrete distribution acting on functions of $\left(n_{j}, n_{p}\right)$ which admit a discrete Fourier transform with respect to these variables and we define the discrete Wigner 'function' $W^{A B}$ as the Fourier transform of $\Omega^{A B}$ with respect to $\left(n_{j}, n_{p}\right)$ :

$$
W_{j, p, k_{j}, k_{p}}^{A B}=\sum_{\left(n_{j}, n_{p}\right) \in Z^{2}} \exp \left(+i k_{j} n_{j}+i k_{p} n_{p}\right) \times\left(\Psi^{A}\right)_{j-n_{j}, p-n_{p}}^{*}\left(\Psi^{B}\right)_{j+n_{j}, p+n_{p}} .
$$

Now we obtain from the equations of motion of the $Q W$, an equation of motion for $W$. Following the computation carried out in the continuous case i.e. for the Dirac equation, we first compute the discrete derivatives of $\Omega^{A B}$ with respect to $j\left(D_{j}\right), p\left(D_{p}\right), n_{j}\left(D_{n j}\right)$ and $n_{p}\left(D_{n p}\right)$, where the derivatives $D_{n j}$ and $D_{n p}$ are defined as $D_{j}$ and $D_{p}$ above. The derivatives of $\Omega$ are best computed using the identities $(12,13)$. One obtains

$\left.\left(D_{j} \Omega^{A B}\right)_{j, p, n_{j}, n_{p}}=\left(D_{j}\left(\Psi^{A}\right)^{*}\right)_{j-n_{j}, p-n_{p}}\left(\Psi^{B}\right)_{j+n_{j}, p+n_{p}}+\left(\Psi^{A}\right)_{j-n_{j}, p-n_{p}}^{*}\left(D_{j} \Psi^{B}\right)\right)_{j+n_{j}, p+n_{p}}+\left(\Delta_{j}^{A B}\right)_{j, p, n_{j}, n_{p}}(25)$, $\left.\left(D_{n j} \Omega^{A B}\right)_{j, p, n_{j}, n_{p}}=-\left(D_{j}\left(\Psi^{A}\right)^{*}\right)_{j-n_{j}, p-n_{p}}\left(\Psi^{B}\right)_{j+n_{j}, p+n_{p}}+\left(\Psi^{A}\right)_{j-n_{j}, p-n_{p}}^{*}\left(D_{j} \Psi^{B}\right)\right)_{j+n_{j}, p+n_{p}}+\left(\Delta_{n j}^{A B}\right)_{j, p, n_{j}, n_{p}}$

Where $\quad\left(\Delta_{j}^{A B}\right)_{j, p, n_{j}, n_{p}}=\left(D_{j}\left(\Psi^{A}\right)^{*}\right)_{j-n_{j}, p-n_{p}}\left(D_{j j} \Psi^{B}\right)_{j-n_{j}, p-n_{p}}+\left(D_{j j}\left(\Psi^{A}\right)^{*}\right)_{j-n_{j}, p-n_{p}}\left(D_{j} \Psi^{B}\right)_{j+n_{j}, p+n_{p}}$

$\left(\Delta_{j}^{A B}\right)_{j, p, k_{\alpha}, k_{\beta}}=-\left(D_{j}\left(\Psi^{A}\right)^{*}\right)_{j-n_{j}, p-n_{p}}\left(D_{j j} \Psi^{B}\right)_{j+n_{j}, p+n_{p}}+\left(D_{j j}\left(\Psi^{A}\right)^{*}\right)_{j-n_{j}, p-n_{p}}\left(D_{j} \Psi^{B}\right)_{j+n_{j}, p+n_{p}} \quad$ (28). Similar relations can be written for $D_{p}$ and $D_{n p}$. Putting all this together delivers $\left(\left(D_{j}+D_{n j}\right) \Omega^{A B}\right)_{j, p, n_{j}, n_{p}}=$ $2\left(\Psi^{A}\right)^{*}{ }_{j-n_{j}, p-n_{p}}\left(D_{j} \Psi^{B}\right)_{j+n_{j}, p+n_{p}}+2\left(D_{j j} \Psi^{A}\right)_{j-n_{j}, p-n_{p}}^{*}\left(D_{j} \Psi^{B}\right)_{j+n_{j}, p+n_{p}} \quad$ (29). And $\left(\left(D_{p}+D_{n_{p}}\right) \Omega^{A B}\right)_{j, p, n_{j}, n_{p}}=$ 
$2\left(\Psi^{A}\right)^{*}{ }_{j-n_{j}, p-n_{p}}\left(D_{p} \Psi^{B}\right)_{j+n_{j}, p+n_{p}}+2\left(D_{p p} \Psi^{A}\right)_{j-n_{j}, p-n_{p}}^{*}\left(D_{p} \Psi^{B}\right)_{j+n_{j}, p+n_{p}}$ (30). Using the equation of motion (11) leads to $\left.\left(\left(D_{j}+D_{n j}\right) \Omega^{A B}\right)_{j, p, n_{j}, n_{p}}-\left(U \sigma_{3}\right)_{C}^{B}\left(D_{p}+D_{n p}\right) \Omega^{A C}\right)_{j, p, n_{j}, n_{p}}=2\left(\Psi^{A}\right)^{*}{ }_{j-n_{j}, p-n_{p}}(U-1)_{C}^{B}\left(\Psi^{C}\right)_{j+n_{j}, p+n_{p}}-$ $\left.2\left(\Psi^{A}\right)_{j-n_{j}, p-n_{p}}^{*}\left(\left(\delta_{C}^{B} D_{j j}-U_{C}^{B} D_{p p}\right)\left(\Psi^{C}\right)\right)_{j+n_{j}, p+n_{p}}+2\left(D_{j j} \Psi^{A}\right)_{j-n_{j}, p-n_{p}}^{*} D_{j}\left(\Psi^{B}\right)\right)_{j+n_{j}, p+n_{p}}-$ $\left.\left.2 D_{p p}\left(\Psi^{A}\right)^{*}\right)_{j-n_{j}, p-n_{p}}\left(U \sigma_{3}\right)_{C}^{B} D_{j}\left(\Psi^{C}\right)\right)_{j+n_{j}, p+n_{p}}$ (31).

Taking the Fourier transform delivers:

$\left(\delta_{C}^{B} D_{j}-\left(U \sigma_{3}\right)_{C}^{B} D_{p}\right) W^{A C}=K^{A B}[\Omega]+M^{A B} \quad(32)$. Where $\quad\left(K^{A B}[\Omega]\right)_{j, p, k_{j}, k_{p}}=\sum_{\left(n_{j}, n_{p}\right) \in Z^{2}} \exp \left(+i k_{j} n_{j}+i k_{p} n_{p}\right) \times$ $\left.\delta_{C}^{B} D_{n j}-\left(U \sigma_{3}\right)_{C}^{B} D_{n p}\right) \Omega_{j, p, n_{j}, n_{p}}^{A C} \quad$ (33). And $\quad\left(M^{A B}\right)_{j, p, k_{j}, k_{p}}=\left(M_{C}^{A B}(W)\right)_{j, p, k_{j}, k_{p}}+\left(M_{S}^{A B}\right)_{j, p, k_{j}, k_{p}} \quad$ (34). With $\left(M_{C}^{A B}(W)\right)_{j, p, k_{j}, k_{p}}=2(\mathrm{U}-1)_{C}^{B}\left(W^{A C}\right)_{j, p, k_{j}, k_{p}}, \quad$ (35), $\quad\left(M_{S}^{A B}\right)_{j, p, k_{j}, k_{p}}=\sum_{\left(n_{j}, n_{p}\right) \in Z^{2}} \exp \left(+i k_{j} n_{j}+i k_{p} n_{p}\right) \mu_{j, p, n_{j}, n_{p}} \quad$ (36), $\left.\mu_{j, p, n_{j}, n_{p}}=-2\left(\Psi^{A}\right)_{j-n_{j}, p-n_{p}}^{*}\left(\left(\delta_{C}^{B} D_{j j}-U_{C}^{B} D_{p p}\right)\right)\left(\Psi^{C}\right)\right)_{j+n_{j}, p+n_{p}}+2\left(D_{j j}\left(\Psi^{A}\right)^{*}\right)_{j-n_{j}, p-n_{p}}\left(D_{j}\left(\Psi^{B}\right)\right)_{j+n_{j}, p+n_{p}}-$ $2\left(D_{p p}\left(\Psi^{A}\right)^{*}\right)_{j-n_{j}, p-n_{p}}\left(U \sigma_{3}\right)_{C}^{B}\left(D_{j}\left(\Psi^{C}\right)\right)_{j+n_{j}, p+n_{p}}$ (37).

The quantity $K^{A B}[\Omega]$ involves discrete Fourier transforms of discrete time- and space-derivatives. To rewrite these into a more appealing form, we use the computation carried out in the previous section. One finds:

$\left(K^{A B}[\Omega]\right)_{j, p, k_{j}, k_{p}}=\sum_{\left(n_{j}, n_{p}\right) \in Z^{2}} \exp \left(+i k_{j} n_{j}+i k_{p} n_{p}\right) \times\left(-i\left(\sin k_{j}\right) \delta_{C}^{B}+i\left(\sin k_{p}\right)\left(U \sigma_{3}\right)_{C}^{B}\right) \Omega_{j, p, n_{j}, n_{p}}^{A C}=-i\left(\left(\sin k_{j}\right) \delta_{C}^{B}-\right.$ $\left.\left(\sin k_{p}\right)\left(U \sigma_{3}\right)_{C}^{B}\right) W_{j, p, k_{j}, k_{p}}^{A C}=\left(K^{A B}(W)\right)_{j, p, k_{j}, k_{p}}(38)$. The final form of the discrete evolution equation obeyed by $W$ is thus:

$\left(\delta_{C}^{B} D_{j}-\left(U \sigma_{3}\right)_{C}^{B} D_{p}\right) W^{A C}-K^{A B}(W)-M_{C}^{A B}(W)=M_{S}^{A B}(39)$.

\subsection{Corrections to the Continuous Transport Equation}

Let us show how to perform an expansion around the continuum limit by computing the lowest order corrections to the transport equation induced by the discreteness of the space-time lattice.

At second order in, the operator $U(\vartheta)$ reads $U(\theta) \sim\left(\begin{array}{cc}1-\frac{\epsilon^{2} m^{2}}{2} & -i \epsilon m \\ -i \epsilon m & 1-\frac{\epsilon^{2} m^{2}}{2}\end{array}\right)$ (40), where designates equality at second order. Since $D_{p}=\epsilon \partial_{x}$, one finds that $U(\theta) \sigma_{3} D_{p} \sim \epsilon\left(\begin{array}{cc}1 & +i \epsilon m \\ -i \epsilon m & -1\end{array}\right) \partial_{x} \quad(41)$, leading to $\delta_{B}^{A} D_{j}-\left(U \sigma_{3}\right)_{B}^{A} D_{p} \sim \epsilon\left(\delta_{B}^{A} \partial_{t}-\left(\sigma_{3}\right)_{B}^{A} \partial_{x}-\right.$ $\epsilon^{2} m\left(\sigma_{2}\right)_{B}^{A} \partial_{x}(42)$, where $\sigma_{2}$ is the second Pauli matrix.

Since $k_{j}=\epsilon k_{t}$ and $k_{p}=\epsilon k_{x}, K^{A B}(W) \sim-i \epsilon\left(k_{j} \delta_{C}^{B}-k_{p}\left(\sigma_{3}\right)_{C}^{B}+\epsilon m k_{p}\left(\sigma_{2}\right)_{C}^{B}\right) W^{A C}$ (43). The first part of the mass term reads $M_{C}^{A B}(W) \sim-i \epsilon m\left(\sigma_{1}\right)_{C}^{B} W^{A C}-\frac{\epsilon^{2} m^{2}}{2} W^{A B}$ (44).

The other part of the mass term depends linearly on the second derivatives $D_{j j}$ and $D_{p p}$. To compute these at second order in $\epsilon$, one can use the first order expressions of $D_{j}$ and $D_{p}$, which are $D_{j} \sim \epsilon \partial_{j}$ and $D_{p} \sim \epsilon \partial_{p}$, combined with the zeroth order expression for $U$, which is $U \sim 1$. Using then the Dirac equation to eliminate the second derivatives leads to:

$M_{S}^{A B} \sim+2 \epsilon^{2} m^{2} W^{A B}(45)$. Including the lowest order corrections to the continuous case, the transport equation obeyed by $W$ thus reads:

$$
\left[\left(\partial_{t}+i k_{j}\right) \delta_{C}^{B}-\left(\partial_{x}+i k_{p}\right)\left(\sigma_{3}\right)_{C}^{B}+i m\left(\sigma_{1}\right)_{C}^{B}\right] W^{A C}=\epsilon\left[m\left(\partial_{x}-i k_{p}\right)\left(\sigma_{2}\right)_{C}^{B}+\frac{3 m^{2}}{2} \delta_{C}^{B}\right] W^{A C}
$$

(46). This equation can be rewritten as $\left[K_{j}\left(\gamma^{j}\right)_{C}^{B}+K_{p}\left(\gamma^{p}\right)_{C}^{B}-m \delta_{C}^{B}\right] W^{A C}=-i \epsilon\left[m K_{p}^{-}\left(\sigma_{3}\right)_{C}^{B}+\right.$ $\left.\frac{3 m^{2}}{2}\left(\sigma_{1}\right)_{C}^{B}\right] W^{A C}(47)$, where $K_{j}=k_{j}-i \partial_{t}, K_{p}=k_{p}-i \partial_{x}, K_{p}^{-}=k_{p}+i \partial_{x}$, and the Dirac matrices satisfying the $2 D$ Clifford algebra are $\gamma^{j}=\sigma_{1}$ and $\gamma^{p}=i \sigma_{2}$. Now, contract (47) on the left with $\left[K_{j}\left(\gamma^{0}\right)_{B}^{D}+\right.$ $\left.K_{p}\left(\gamma^{1}\right)_{B}^{D}-m \delta_{B}^{D}\right]$.This delivers an equation of the form $(M+i K)_{C}^{D} W^{A C}=0$ where both $M$ and $K$ 
are hermitian. One thus deduces that both $M_{C}^{D} W^{A C}$ and $K_{C}^{D} W^{A C}$ vanish. The explicit computation delivers $M=M_{0}+\epsilon M_{1}, K=K_{0}+\epsilon K_{1}$ (48). With $M_{0}=k_{j}^{2}-k_{p-}^{2} m^{2}-\left(\partial_{t}^{2}-\partial_{x}^{2}\right)$ (49), $M_{1}=m\left(k_{j} k_{p}+\partial_{t} \partial_{x}\right) \sigma_{2}+\frac{3 m^{2}}{2} \partial_{t} 1+\partial_{x} \sigma_{3}$ (50), $K_{0}=-2 k_{j} \partial_{t}+2 k_{p} \partial_{x}$ (51), $K_{1}=-m\left(k_{j} \partial_{x}-\right.$ $\left.k_{p} \partial_{t}\right) \sigma_{2}-m\left(k_{p}^{2}+\partial_{x}^{2}\right) \sigma_{1}+\frac{3 m^{2}}{2} k_{j} 1+k_{p} \sigma_{3}(52)$.

It is clear that the equation $M W=0$ is a mass-shell condition (hence the notation $M$ ) on the Wigner function $W$. Observe that, even for vanishing i.e. for the Dirac equation, the usual massshell condition $k_{j}^{2}-k_{p}^{2}=m^{2}$ is not exact, but only an approximation valid for Wigner functions that varies sufficiently slowly in both space and time. The $M_{1}$ term represents the correction to the mass-shell equation at the first order in the finite step of the lattice over which the DTQW is defined.

In order to understand the second equation, $K W=0$, let us recall that, in flat $2 D$ space-times, one particle relativistic non quantum transport can be described by introducing a scalar distribution $f$ in $4 D$ phase-space $R^{4}=\left\{\left(t, x, p^{t}, p^{x}\right)\right\}$ which verifies the equations in the form $p^{\mu} \frac{\partial f}{\partial x^{\mu}}+\frac{\partial}{\partial p^{\mu}}\left(F^{\mu} f\right)+\frac{\partial^{2}}{\partial p^{\mu} \partial p^{v}}\left(D^{\mu v} f\right)=C(f, f)(53)$. For example, in the relativistic Boltzmann or Boltzmann-Vlasov equations [51-53], $F$ is the force that acts on the particles, $D$ vanishes identically and $C(f, f)$ is the relativistic Boltzmann collision kernel. For relativistic stochastic processes [54], the transport equation is the so-called Kolmogorov equation (hence the notation $\mathrm{K}$ ). For these processes, $C(f, f)$ vanishes, $F$ represents the deterministic part of the force acting on the particles, and $D$ is the squared amplitude of a Gaussian noise.

Now we consider the free Dirac fermions, it is natural to set $F=0$. Since there is no noise in the problem considered, $D$ also vanishes, and so does $C(f, f)$, because the one fermion under consideration does not collide with other fermions. The linear operator acting on $f$ in the nonquantum transport equation then coincides formally with $K_{0}$ and describes the Dirac Hamiltonian flow in $4 D$ relativistic space-time. The operator $K_{1}$ determines the correction induced by the finite lattice-step. If noise is added to the problem, extra terms involving at least the first and second order derivatives with respect to $k$ would appear in the transport equation, making it a quantum discrete Kolmogorov equation for fermions.

\section{Discussion}

We have defined a relativistic Wigner function for free DTQWs on the square $2 D$ space-time lattice. This definition uses the concepts of discrete derivatives and distributions which have also been introduced. We have established the transport equation that is obeyed by the relativistic Wigner function and proved that this equation degenerates in the continuum limit into the transport equation that is obeyed by the Wigner function of Dirac fermions. We have finally computed the first corrections to this equation induced by the discreteness of the lattice and discussed how this equation connects with non-quantum relativistic transport in $4 D$ phase-space.

It is intuitive to discuss how these results compare with others, such as non manifestly covariant definitions of relativistic Wigner functions. Let us focus for example on [55], which introduces non manifestly covariant Wigner functions for Dirac and Maxwell fields in the context of relativistic Quantum Field Theory (QFT). First, QFT is a quantum theory of fields, not of particles. In particular, the classical coordinates are classical fields, not positions in space-time, and the momenta are also fields. It may, therefore, appear unnatural to try and define Wigner functions 
for QFT which depend on coordinates in classical phase-space, and a more natural option would be to introduce Wigner 'functions' which are functionals of the fields and of their conjugate momenta. This route is certainly not trivial, but we believe it can produce relativistically covariant Wigner functionals. Leaving this to future publications, let us now suppose one nevertheless wants to construct, for relativistic QFT or for an approximate 'relativistic quantum mechanics', Wigner functions defined over a one-particle relativistic phase-space. Classical relativistic statistical physics offers several choices for the one particle phase-space. A first choice is the tangent bundle of the space-time manifold. A second choice is the cotangent bundle. In both cases, the phasespace has twice the dimension of the space-time manifold. Now, a classical particle lives on its mass-shell. One can therefore choose as classical relativistic phase-space the massshell bundle. At any given point in a $D$-dimensional space-time, the mass-shell is (one sheet of) a $D-1$ dimensional hyperboloid in momentum space and the mass-shell bundle is thus of dimension $2 D-$ 1.

All three choices are equivalent in classical statistical physics $[52,53]$. The transport equations obtained by choosing the tangent or the cotangent bundle as phase-space look simpler than those obtained by choosing the mass-shell bundle. But the dynamics encoded in these simpler-looking equations leave particles on their mass-shells, so restricting these equations to the mass-shells delivers the transport equations obtained by working directly with the mass-shell bundles. Because the associated transport equations look simpler, most authors working in classical relativistic statistical physics now choose to work in the tangent or cotangent bundle. Working with the mass-shell bundle may appear more natural in situations where the problem presents a preferred reference frame, as is the case for example with relativistic stochastic processes [54]. But, even in these cases, rewriting everything in a manifestly covariant manner by working in the tangent or cotangent bundle is interesting.

Things are more complex in quantum physics. The choice between working in a manifestly covariant manner in the tangent or cotangent bundle and working in a non manifestly covariant manner in a phase-space of lower dimension remains. However, both options now are not equivalent (in the mathematical sense). Consider for example the $2 D$ Dirac equation. As shown by the equation $M W=0$ derived and discussed in the previous section, the momentum $p$ which appears as a variable of the Wigner function defined in a manifestly covariant treatment is not restricted to the classical mass-shell [47], nor to any hyper-surface in momentum space, even, which corresponds to $2 D$ continuous Dirac fermions. The manifestly covariant treatment is thus not equivalent to a non manifestly covariant treatment developed in a phase-space of lower dimension. For the Dirac equation, both treatments exist. Indeed, references [47-49] use a manifestly covariant approach while reference [55] uses a non manifestly covariant one. This author believes that there are good reasons to stick to a manifestly covariant treatment, at least when no preferred reference frame exists, as is the case for the Dirac equation. For example, the fermionic transport equation obtained in [55] is much more complex than the transport equation obtained or used in [47-49] It, therefore, does not seem to admit a simple geometrical interpretation in terms of Hamiltonian flow in phase-space or mass-shell violation, making, in particular, the connexion to non-quantum transport hard to analyze. And changing reference frame in non-covariant transport equations is a really arduous task, as explicitly noted in [55].

Now QWs defined on a grid do exhibit a preferred reference frame, and this is why several authors have presented a non manifestly covariant construction of Wigner functions for QWs. The 
drawback of this approach is seen when one considers QWs which admit a continuous limit identical to the Dirac equation. The transport equation derived from a non manifestly covariant treatment does not admit as continuous limit the standard transport equation obeyed by the manifestly covariant Wigner function of the Dirac field, but rather the transport equation derived in [55]. As explained in the previous paragraph, this non manifestly covariant equation does not resemble classical relativistic transport equations and is, therefore, ill suited to study decoherence for these walks. We feel this is a serious drawback, especially because decoherence is a crucial issue in quantum information.

The results presented in this article can be extended in several directions. One should first address DTQWs defined, both on higher dimensional and on more general lattices, like for example planar triangular and hexagonal ones [23, 24]. This could be done by using Fourier series defined on general spectral sets (see for example [56] and references therein). An extension to DTQWs defined on graphs should also be envisaged [57-59]. Since several DTQWs with non constant mixing operators can be interpreted as fermions coupled to discrete gauge fields [36-40, 60-65], one should define a Wigner function which incorporates these gauge fields and, in particular the electromagnetic field and the gravitational field. For example, defining a gaugeinvariant Wigner function for Dirac particles couples to electromagnetic fields is highly non trivial [47] and one wonders how the problem translates to DTQWs. Finally, DTQWs defined through unitaries that present a time randomness decohere and behave asymptotically like non-quantum diffusions (see for example [66] and references therein). One then expects the transport equation for the relativistic Wigner function to approach asymptotically relativistic transport equations similar to those obtained for relativistic stochastic processes [54,67]. This should be confirmed and the asymptotics fully analyzed.

\section{Author Contributions}

Fabrice Debbasch did all the work.

\section{Competing Interests}

The author has declared that no competing interests exist.

\section{References}

1. Feynman RP, Hibbs AR, Styer DF. Quantum mechanics and path integrals. International Series in Pure and Applied Physics. McGraw-Hill Book Company. 1965.

2. GrÖssing G, Zeilinger A. Quantum cellular automata. Compl Syst. 1988; 2: 197-208.

3. Aharonov Y, Davidovich L, Zagury N. Quantum random walks. Phys Rev A. 1993; 48: 1687.

4. Meyers DAT. From quantum cellular automata to quantum lattice gases. J Stat Phys. 1996; 85: 551-574.

5. Schmitz H, Matjeschk R, Schneider $\mathrm{CH}$, Glueckert J, Enderlein $\mathrm{M}$, Huber T, et al. Quantum walk of a trapped ion in phase space. Phys Rev Lett. 2009; 103: 090504.

6. Zahringer F, Kirchmair G, Gerritsma R, Solano E, Blatt R, Roos CF. Realization of a quantum walk with one and two trapped ions. Phys Rev Lett. 2010; 104: 100503. 
7. Schreiber A, Cassemiro KN, Potocek V, Gabris A, Mosley PJ, Andersson E, et al. Photons walking the line: A quantum walk with adjustable coin operations. Phys Rev Lett. 2010; 104: 050502.

8. Karski M, Forster L, Cho JM, Steffen A, Alt W, Meschede D, et al. Quantum walk in position space with single optically trapped atoms. Science. 2009; 325: 174-177.

9. Genske M, Alt W, Steffen A, Werner AH, Werner RF, Meschede D, et al. Electric quantum walks with individual atoms. Phys Rev Lett. 2013; 110: 190601

10. Sansoni L, Sciarrino F, Vallone G, Mataloni P, Crespi A, Ramponi R, et al. Two-particle bosonicfermionic quantum walk via integrated photonics. Phys Rev Lett. 2012; 108: 010502.

11. Sanders BC, Bartlett SD, Tregenna B, Knight PL. Quantum quincunx in cavity quantum electrodynamics. Phys Rev A. 2003; 67: 042305.

12. Zhi-Hao B, Hao Q, Xiang Z, Jian L, Peng X. A quantum walk in phase space with resonatorassisted double quantum dots. Chinese Phys B. 2016; 25: 020307.

13. Wan LX, Yan LB, Huang JG, Zhang G, Kwek LC, Fitzsimons J, et al. A large-number and multilayer quantum walk using silicon nano-photonic chip. In Conference on Lasers and Electro-Optics, OSA Terchnical Digest (online); Optical Society of America, 2018.

14. Perets HB, Lahini Y, Pozzi F, Sorel M, Morandotti R, Silberberg Y. Realization of quantum walks with negligible decoherence in waveguide lattices. Phys Rev Lett. 2008; 100: 170506.

15. Ambainis A. Quantum walk algorithm for element distinctness. SIAM J Comput. 2007; 37: 210239.

16. Magniez F, Nayak A, Roland J, Santha M. Search via quantum walk. In SIAM J. Comput Proceedings of the thirty-ninth annual ACM symposium on Theory of computing; New York, 2007; 575-584.

17. Aslangul C. Quantum dynamics of a particle with a spin-dependent velocity. J Phys A. 2005; 38: 1-16.

18. Bose S. Quantum communication through an unmodulated spin chain. Phys Rev Lett. 2003; 91 : 207901.

19. Burgarth D. Quantum state transfer with spin chains. PhD Thesis. 2006.

20. Bose S. Quantum communication through spin chain dynamics: An Introductory Overview. Contemp Phys. 2007; 48: 13-30.

21. Di Molfetta G, Debbasch F, Brachet M. Non-linear optical Galton board: Thermalization and continuous limit. Phys Rev E. 2015; 92: 042923.

22. Bougroura $H$, Aissaoui $H$, Chancellor N, Kendon V. Quantum walk transport properties on graphene structures. Phys Rev A. 2016; 94: 062331.

23. Arrighi $P$, Di Molfetta $G, M^{\prime}$ arquez Mart'ın I, $P^{\prime}$ erez $A$. Dirac equation as a quantum walk over the honeycomb and triangular lattices. Phys Rev A. 2018; 97: 062111.

24. Jay G, Debbasch F, Wang JB. Dirac quantum walks on triangular and honeycomb lattices. Phys Rev A. 2019; 99: 032113.

25. Hatifi M, Di Molfetta G, Debbasch F, Brachet M. Quantum walk hydrodynamics. Scient Rep. 2019; 9: 2989.

26. Collini E, Wong CY, Wilk KE, Curmi PMG, Brumer P, Scholes GD. Coherently wired lightharvesting in photosynthetic marine algae at ambient temperature. Nature. 2010; 463: 644647. 
27. Engel GS, Calhoun TR, Read RL, Ahn TK, Manal T, Cheng YC, et al. Evidence for wavelike energy transfer through quantum coherence in photosynthetic systems. Nature. 2007; 446: 782-786.

28. Strauch FW. Relativistic quantum walks. Phys Rev A. 2006; 73: 054302.

29. Strauch FW. Relativistic effects and rigorous limits for discrete- and continuous-time quantum walks. J Math Phys. 2007; 48: 082102.

30. Bialynicki-Birula I. Weyl, Dirac, and Maxwell equations on a lattice as unitary cellular automata. Phys Rev D. 1994; 49: 6920.

31. Yepez J. Relativistic path integral as a lattice-based quantum algorithm. Quantum Inf Proc. 2005; 4: 471-509.

32. Arrighi P., Nesme V. and Forets M. The Dirac equation as a quantum walk: Higher dimensions, observational convergence. J Phys A. 2014; 47: 465302.

33. Bisio A, D'Ariano G, Tosini A. Quantum field as a quantum cellular automaton: The Dirac free evolution in one dimension. Ann Phys. 2015; 354: 244-264.

34. Bisio A, D’Ariano G, Perinotti P, Tosini A. Weyl, Dirac and Maxwell quantum cellular automata. Found Phys. 2015; 45: 1203-1221.

35. D'Ariano G, Erba M, Perinotti P. Isotropic quantum walks on lattices and the Weyl equation. Phys Rev A. 2017; 96: 062101.

36. Cedzich C, Ryba'r T, Werner AH, Alberti A, Genske M, Werner RF. Propagation of quantum walks in electric fields. Phys Rev Lett. 2013; 111: 160601.

37. Di Molfetta G, Brachet M, Debbasch F. Quantum walks as massless Dirac fermions in curved space-time. Phys Rev A. 2013; 88: 042301.

38. Di Molfetta G, Brachet M, Debbasch F. Quantum walks in artificial electric and gravitational Fields. Physica A. 2008; 397: 157-168.

39. Arnault P, Debbasch F. Quantum walks and discrete gauge theories. Phys Rev A. 2016; 93 : 052301.

40. Arnault P, Di Molfetta G, Brachet M, Debbasch F. Quantum walks and non-abelian discrete gauge theories. Phys Rev A. 2016; 94: 01233.

41. Curtright TL, Fairlie DB, Zachos ZK. A concise treatise on quantum mechanics in phase space. World Sci Pub Co Inc, 2014.

42. de Gosson M, Luef F. Moyal bracket and ehrenfest's theorem in born-jordan quantization. Quantum Rep. 2019; 1: 71-81.

43. Hinarejos $M, P^{\prime}$ erez $A$, Banuls $M C$. Wigner function for a particle in an infinite lattice. New J Phys. 2012; 14: 103009.

44. Alberti A, Alt W, Werner R, Meschede D. Decoherence models for discrete-time quantum walks and their application to neutral atom experiments. New J Phys. 2014; 16: 123052.

45. Hinajeros $M$, Banuls MC, Perez A. Wigner formalism for a particle on an infinite lattice: Dynamics and spin. New J Phys. 2015; 17: 013037.

46. Hinajeros $M$, Banuls $M C$, Perez A. A study of wigner functions for discrete-time quantum walks. J Comput Theoret Nanosc. 2013; 10: 1626.

47. Vasak D, Gyulassy M, Helze HTh. Quantum transport theory for Abelian plasmas. Ann Phys. 1987; 173: 462-492.

48. Helze HTh, Gyulassy M, Vasak D. Transport equations for the QCD quark Wigner operator. Nucl Phys B. 1986; 276: 706-728. 
49. Gao J, Pu S, Wang Q. Covariant chiral kinetic equation in Wigner function approach. Phys Rev D. 2017; 96: 016002.

50. Dean DS, Le Doussal P, Majumdar SN, Sher G. Wigner function of noninteracting trapped fermions. Phys Rev A. 2018; 97: 063614.

51. Israel W. Covariant fluid mechanics and thermodynamics: An introduction, in Relativistic Fluid Dynamics, A.M. Anile and Y. Choquet-Bruhat, eds, Lecture Notes in Mathematics 1385, 1987.

52. Debbasch F, van Leeuwen W. General relativistic Boltzmann equation I: Covariant treatment. Physica A. 2009; 388: 1079-1104.

53. Debbasch F, van Leeuwen W. General relativistic Boltzmann equation II: Manifestly covariant treatment. Phys A. 2009; 388: 1818-1834.

54. Chevalier C, Debbasch F. Relativistic diffusions: A unifying approach. J Math Phys. 2008; 49: 043303.

55. Bialynicki-Birula I. Relativistic Wigner functions. EPJ Web of Conferences. 2014; 78.

56. Xu Y. Fourier series and approximation on hexagonal and triangular domains. Constr Approx. 2010; 31: 115.

57. Bru LA, de Valc'arcel GJ, Di Molfetta G, P'erez A, Rolda`n E, Silva F. Quantum walk on a cylinder. Phys Rev A. 2016; 94: 032328.

58. D’Ariano G, Erba M, Perinotti P, Tosini A. Virtually Abelian quantum walks. J Phys A. 2016; 50: 035301.

59. Bisio A, D'Ariano G, Erba M, Perinotti P, Tosini A. Quantum walks with a one-dimensional coin. Phys Rev A. 2016; 93: 062334.

60. Arrighi P, Facchini S, Forets M. Quantum walking in curved spacetime. Quantum Inf Proc. 2016; 15: 3467-3486.

61. Arnault P, Debbasch F. Landau Levels for discrete time quantum walks in artificial magnetic fields. Phys A. 2016; 443: 179-191.

62. Bru LA, Hinarejos M, Silva F, de Valca'rcel GJ, Rolda'n E. Electric quantum walks in two dimensions. Phys Rev A. 2016; 93: 032333.

63. Arrighi $\mathrm{P}$, Patricot $\mathrm{CH}$. Quantum walking in curved spacetime: $(3+1)$ dimensions, and beyond. Quantum Inf Comput. 2016; 17.

64. Arnault P, Debbasch F. Quantum walks and gravitational waves. Ann Phys. 2017; 383: 645-661.

65. Cedzich C, Geib T, Werner AH, Werner RF. Quantum walks in external gauge fields. J Mathemat Phys. 2019; 60: 012107.

66. Di Molfetta G, Debbasch F. Discrete-time Quantum Walks in random artificial Gauge Fields. Quant Stud Mathem Found. 2016; 3: 293-311.

67. Debbasch F, Mallick K, Rivet JP. Relativistic Ornstein-Uhlenbeck process. J Stat Phys. 1997; 88: 945. 


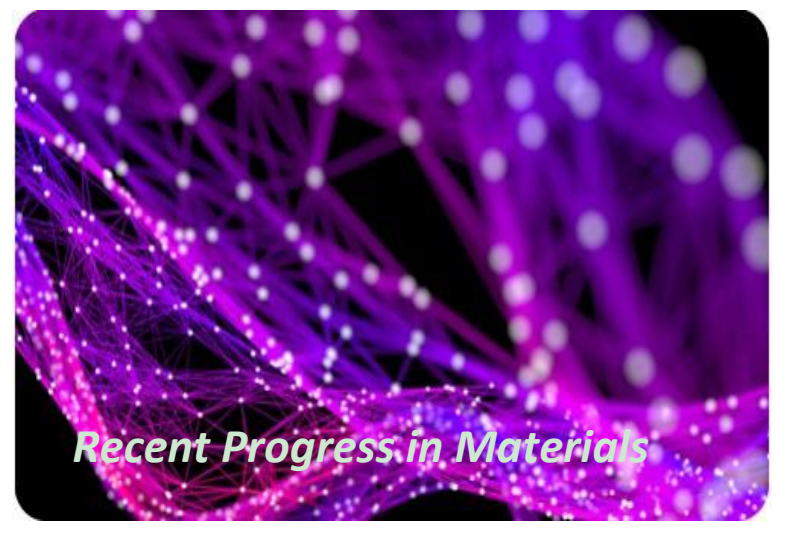

Enjoy Recent Progress in Materials by:

1. Submitting a manuscript

2. Joining in volunteer reviewer bank

3. Joining Editorial Board

4. Guest editing a special issue

For more details, please visit:

http://www.lidsen.com/journals/rpm 